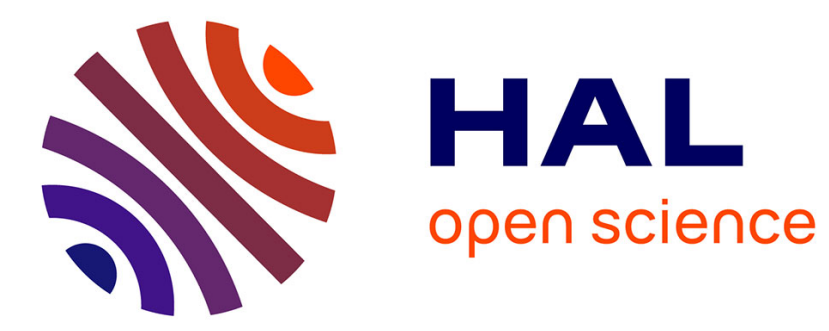

\title{
Mesure simultanée de la vitesse de phase, de l'atténuation des ondes ultrasonores, de l'épaisseur et de la masse volumique de matériaux composites
}

C. Graciet, B. Hosten

\section{- To cite this version:}

C. Graciet, B. Hosten. Mesure simultanée de la vitesse de phase, de l'atténuation des ondes ultrasonores, de l'épaisseur et de la masse volumique de matériaux composites. Journal de Physique IV Proceedings, 1994, 04 (C5), pp.C5-1177-C5-1180. 10.1051/jp4:19945259 . jpa-00252948

\section{HAL Id: jpa-00252948 https://hal.science/jpa-00252948}

Submitted on 1 Jan 1994

HAL is a multi-disciplinary open access archive for the deposit and dissemination of scientific research documents, whether they are published or not. The documents may come from teaching and research institutions in France or abroad, or from public or private research centers.
L'archive ouverte pluridisciplinaire HAL, est destinée au dépôt et à la diffusion de documents scientifiques de niveau recherche, publiés ou non, émanant des établissements d'enseignement et de recherche français ou étrangers, des laboratoires publics ou privés. 


\title{
Mesure simultanée de la vitesse de phase, de l'atténuation des ondes ultrasonores, de l'épaisseur et de la masse volumique de matériaux composites
}

\author{
C. GRACIET et B. HOSTEN
}

Laboratoire de Mécanique Physique, URA 867 du CNRS, Université de Bordeaux I, 351 Cours de la Libération, 33405 Talence cedex, France

\begin{abstract}
Under impact, the geometrical and mechanical properties of materials are modified. In composite materials, the damage is due to delamination between plies and macro-cracking appearance inside the matrix. These induce large modifications in velocity and attenuation of ultrasonic wave. Because thickness and density of the impacted material can also be influenced, we propose a simple ultrasonic method to measure these four properties in the same time. Plate thickness and wave velocity are deduced from the time of fly of several echoes, attenuation and density are obtained from a spectral analysis of these echoes. We test the method on three different materials : glass, PVC, glass/epoxy. The results obtained are quite good. In a further work, we will extend this method to focal probe, to increase spatial resolution.
\end{abstract}

\section{1 - INTRODUCTION.}

L'emploi de matériaux composites soumis à des endommagements tels que choc, fatigue, immersion prolongée implique de pouvoir lier le type d'endommagement à la sollicitation exercée. Plus particulièrement, lors d'un impact sur une plaque de composite, l'endommagement inhomogène qui en résulte est fonction de l'énergie d'impact : fissuration de la matrice puis déchaussement des fibres et enfin délaminage. Dans le but de qualifier puis de quantifier l'endommagement issu d'une telle sollicitation, nous prévoyons de développer un outil d'imagerie quantitative, basée sur la mesure de propriétés mécaniques et physiques du matériau telles que l'épaisseur, la masse volumique, la célérité et l'atténuation des ondes ultrasonores.

La première étape de ce projet, développée dans cet article, consiste à établir une méthode simple de mesure en faisceau plan, puis ultérieurement d'étendre cette technique aux faisceaux focalisés.

De nombreux auteurs ont travaillé sur la mesure de ces caractéristiques pour des plaques fines ou épaisses. Cette grandeur est relative à la longueur d'onde utilisée. Une plaque est épaisse lorsque les longueurs d'onde dans l'impulsion ultrasonore sont suffisamment petites pour permettre la séparation temporelle des échos.

Dans le cadre des plaques minces, Tsukahara [1] détermine le coefficient d'atténuation d'une onde acoustique grâce à la mesure du module du coefficient de réflexion complexe, aux fréquences de résonance. Par ailleurs, Haines [2] examine plus particulièrement le cas d'une plaque mince dont l'une des faces est libre ( sans contrainte ). Par l'analyse de l'amplitude et de la phase du coefficient de réflexion complexe, il en déduit les fréquences de résonance puis la vitesse des ondes, leur atténuation et l'impédance du matériau. Pialucha [3] présente une technique de mesure de la vitesse de phase dans un matériau pouvant être dispersif comme atténuant, reposant sur l'acquisition de l'amplitude des spectres des signaux rétrodiffusés. Kinra \& Dayal [4], grâce à un programmes d'optimisation de la fonction transfert de la plaque, accèdent à la célérité et l'atténuation des ondes ultrasonores dans des matériaux dispersifs ou non. Dans un récent article, Kinra \& Zhu [5] utilisent également l'optimisation de la fonction de transfert afin de déterminer simultanément la célérité des ondes et l'épaisseur de couche mince. 
Les plaques épaisses permettant de travailler dans le domaine temporel, Hsu \& Hughes [6] calculent la célérité des ondes ultrasonores ainsi que l'épaisseur de l'échantillon par la mesure des temps de vol des échos rétro-diffusés aux interfaces. Cette méthode, simple et pratique, n'est cependant pas applicable aux matériaux très dispersifs. Pour pallier à cet inconvénient, d'autres auteurs préfèrent travailler dans le domaine fréquentiel. Daniel [7] détermine l'atténuation des ondes grâce l'acquisition de l'amplitude du spectre des signaux réfléchis. Kinra \& Dayal [4] calculent, grâce à l'amplitude et la phase des spectres, la célérité et l'atténuation des ondes. Dayal [8] analyse la phase des spectres pour en déduire la célérité des ondes ainsi que l'épaisseur de la plaque tout en tenant compte des variations éventuelles d'épaisseur.

Cependant, aucun de ces auteurs ne mesure la totalité de ces 4 grandeurs, évoluant simultanément au cours d'un endommagement. Nous proposons une méthode simple permettant de mesurer simultanément, l'épaisseur et la masse volumique de l'échantillon ainsi que la célérité et l'atténuation des ondes ultrasonores.

\section{2 - THEORIE.}

La configuration dans laquelle nous nous plaçons est représentée sur la figure 1. Le système fonctionne en réflexion -i.e.- un seul transducteur en émission - réception. L'échantillon se présente sous la forme d'une plaque plane épaisse d'épaisseur d et de célérité $c_{1}$. Celle-ci, immergée dans une cuve, est insonifiée par une onde plane longitudinale en incidence normale. Cette onde est successivement réfléchie aux interfaces de l'échantillon puis sur une plaque de verre parfaitement plane servant de réflecteur. La mesure des différentes grandeurs se fera grâce à l'analyse de ces échos.

Soit $s_{0}(t)=f\left(\omega t-k_{0} x\right)$ l'expression du champ de déplacement de l'onde de compression incidente. Les champs de déplacement des différents échos sont donnés par :

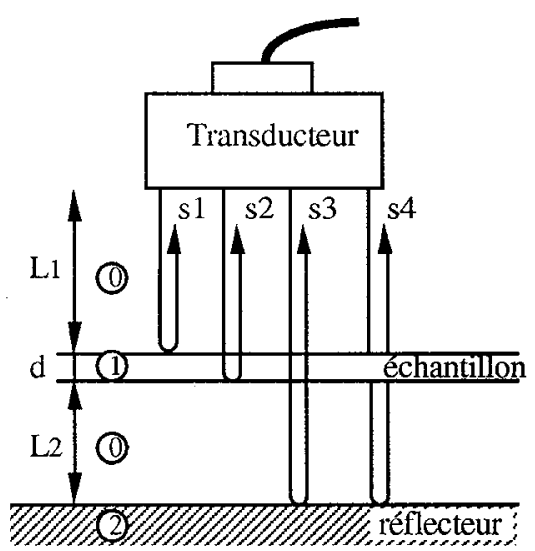

Figure 1: Schéma de principe.

$$
\begin{aligned}
& s_{1}(t)=R_{01} f\left(\omega t-2 k_{0} L_{1}\right) \\
& s_{2}(t)=T_{01} R_{10} T_{10} f\left(\omega t-2 k_{0} L_{1}-2 k_{1} d\right) \\
& s_{3}(t)=T_{01} T_{10} R_{12} T_{01} T_{10} f\left(\omega t-2 k_{0}\left(L_{1}+L_{2}\right)-2 k_{1} d\right) \\
& s_{4}(t)=R_{12} f\left(\omega t-2 k_{0}\left(L_{1}+L_{2}+d\right)\right)
\end{aligned}
$$

relations dans lesquelles $R_{\mathrm{ij}}$ et $T_{\mathrm{ij}}$ représentent les coefficients de réflexion et de transmission du milieu $\mathrm{j}$ dont les expressions en incidence normale sont:

$$
R_{i j}=\frac{Z_{j}-Z_{i}}{Z_{j}+Z_{i}} \text { et } T_{i j}=\frac{2 Z_{j}}{Z_{j}+Z_{i}} \quad Z_{j}=\rho_{j} c_{j}
$$

\section{1 - Mesure de l'épaisseur et de la célérité.}

Nous plaçant dans le cadre des plaques épaisses, les différents échos sont séparés temporellement. La référence [6] détaille plusieurs configurations permettant de mesurer simultanément l'épaisseur de l'échantillon et la célérité des ondes. La méthode basée sur l'acquisition de seulement 3 échos [9], n'est pas applicable pour des matériaux absorbants et pour lesquels il est essentiel de restreindre le nombre de passage dans la plaque. De plus, comme nous le verrons au paragraphe suivant, les mesures de la masse volumique et du coefficient d'atténuation nécessitent l'acquisition des 4 échos.

Les temps de vol des échos $\mathrm{s} 1$ et $\mathrm{s} 2$ sont $\mathrm{t} 1$ et $\mathrm{t} 2$ alors que $\mathrm{t} 3$ et $\mathrm{t} 4$ sont respectivement les temps de vol de l'onde réfléchie sur le réflecteur avec et sans échantillon. 
Dans ces conditions, les expressions de la célérité et de l'épaisseur sont alors [6]:

$$
\mathrm{c}_{1}=\mathrm{c}_{0}\left(\frac{\mathrm{T}_{2}}{\mathrm{~T}_{1}}+1\right) \quad \text { (3) } \quad ; \quad \mathrm{d}=\frac{\mathrm{c}_{0}}{2}\left(\mathrm{~T}_{2}+\mathrm{T}_{1}\right)
$$

avec $T_{1}=t_{2}-t_{1} \quad$ et $\quad T_{2}=t_{3}-t_{4}$.

\section{2 - Mesure de l'atténuation et de la masse volumique.}

La célérité et l'épaisseur étant maintenant des quantités connues, les 2 autres grandeurs se déduisent des mesures d'amplitudes.

Soit $S^{*}(v)$ la transformée de fourier de $s(t)$, l'amplitude de l'écho i est définie relativement à l'écho de référence 4 :

$$
A_{i}=\frac{\left|S_{i}^{*}\left(v_{0}\right)\right|}{\left|S_{4}^{*}\left(v_{0}\right)\right|} \quad \text { à la fréquence } v_{0}
$$

$R_{02}$ étant le coefficient de réflexion de la plaque de verre ( réflecteur), la masse volumique et le coefficient d'atténuation sont donnés par :

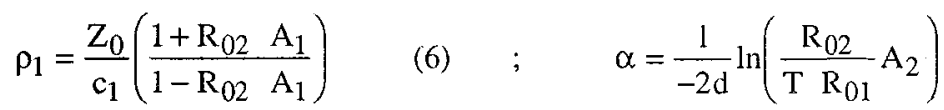

avec $\mathrm{T}=\mathrm{T}_{01} \mathrm{~T}_{10}$ le coefficient de transmission des 2 interfaces.

\section{3 - RESULTATS.}

Afin de valider la méthode, nous avons réalisé une série de mesures sur divers matériaux ; une lame de verre, grande impédance et atténuation nulle, une lame de PVC, faible impédance et atténuation non nulle et enfin un composite Verre/Epoxyde $\left[0_{2}, 90_{6}, 0_{2}\right]$, matériau anisotrope d'impédance proche de celle du PVC, suivant la normale à l'échantillon. L'acquisition des signaux est moyennée 10 fois. Les mesures ont été réalisées en 40 positions, à l'aide d'un transducteur plan large bande de fréquence centrale $4,69 \mathrm{MHz}$. Le choix de la fréquence est dicté par la volonté de travailler dans le cadre des plaques épaisses tout en considérant le composite homogène. Les moyennes de ces mesures ainsi que leurs variations maximales sont reportées dans le tableaux 1 .

Tableau 1 : Récapitulatif des résultats

\begin{tabular}{|l|l|c|c|c|}
\hline & & Verre & PVC & Verre/Epoxyde \\
\hline $\begin{array}{l}\text { célérité } \\
(\mathrm{mm} / \mu \mathrm{s})\end{array}$ & référence & 5,87 & 2,36 & 3,00 \\
\cline { 2 - 5 } & mesurée & $5,85 \pm 0,01$ & $2,35 \pm 0,001$ & $3,00 \pm 0,01$ \\
\hline $\begin{array}{l}\text { épaisseur } \\
(\mathrm{mm})\end{array}$ & rétérence & 3,86 & 10,03 & 1,82 \\
\cline { 2 - 5 } & mesurée & $3,86 \pm 0,005$ & $10,02 \pm 0,01$ & $1,81 \pm 0,006$ \\
\hline $\begin{array}{l}\text { masse volumique } \\
\left(\mathrm{gr} / \mathrm{cm}^{3}\right)\end{array}$ & référence & 2,51 & 1,49 & 1,80 \\
\cline { 2 - 5 } & mesurée & $2,54 \pm 0,07$ & $1,46 \pm 0,007$ & $1,90 \pm \pm 0,05$ \\
\hline $\begin{array}{l}\text { atténuation } \\
(\mathrm{Np} / \mathrm{mm})\end{array}$ & référence & 0,004 & 0,115 & 0,325 \\
\cline { 2 - 5 } & mesurée & $0,007 \pm 0,002$ & $0,115 \pm 0,002$ & $0,29 \pm 0,04$ \\
\hline
\end{tabular}

Les valeurs de référence sont obtenues traditionnellement : l'épaisseur est mesurée grâce à un micromètre $( \pm 0,01 \mathrm{~mm})$, la masse volumique à l'aide d'une double pesée $( \pm 0,02)$, la célérité et l'atténuation à l'aide du banc ultrasonore en transmission de la référence [9].

Les mesures des temps de vol sont obtenues par corrélation des différents échos par rapport à l'écho de référence $\mathrm{s} 4$. Les matériaux testés n'étant pas trop atténuants, la forme des signaux est peu modifiée après le passage dans l'échantillon. Les fluctuations dans les mesures d'épaisseur et de célérité sont dues aux seules erreurs de mesure des temps de vol. A partir des formules (3), (4), (6) et (7), les calculs d'erreur sur l'épaisseur, la célérité, la masse volumique et l'atténuation, dans lesquels sont introduites les moyennes précédentes, foumit le tableau 2. Dans ces calculs interviennent une erreur absolue sur la mesure des temps 
de vol, identifiée à la période d'échantillonnage i.e. $\Delta \mathrm{T}=0,01 \mu \mathrm{s}$, et unè erreur relative sur la mesure d'amplitudes estimée à $5 \%$.

Tableau 2 : Erreurs absolues calculées .

\begin{tabular}{|l|c|c|c|}
\hline & Verre & PVC & Verre/Epoxyde \\
\hline$\Delta$ célérité & 0,08 & 0,004 & 0,004 \\
\hline$\Delta$ épaisseur & 0,02 & 0,02 & 0,02 \\
\hline$\Delta$ masse volumique & 0,6 & 0,07 & 0,2 \\
\hline$\Delta$ atténuation & $/$ & 0,004 & 0,01 \\
\hline
\end{tabular}

Pour la lame de verre, célérité et épaisseur sont proches des valeurs de référence, leurs variations de $0,12 \%$ sont inférieures aux erreurs de mesure calculées. La masse volumique est proche de la valeur obtenue par la pesée mais présente des variations impottantes; l'erreur sur cette grandeur est liée à l'erreur de mesure sur l'amplitude par la relation :

$$
\frac{\Delta \rho_{1}}{\rho_{1}}=\frac{2 R_{02}}{1-\left(A_{1} R_{02}\right)^{2}} \Delta A_{1}
$$

On note alors que l'erreur sur la détermination de $\rho_{1}$ est inversement proportionnelle à l'amplitude relative $A_{1}$. Cette technique sera donc mieux adaptée à l'étude des matériaux de faible impédance tels que PVC, résines, composites à matrice polymérique. L'atténuation dans le verre étant théoriquement nulle, la moindre variation lors de l'acquisition de l'amplitude génère des erreurs de $25 \%$.

Les mesures sur l'échantillon de PVC confirment les remarques précédentes : célérité, épaisseur et masse volumique sont en accord avec les valeurs théoriques. L'atténuation présente de faibles variations, tout en ayant une valeur moyenne correcte, ce qui conforte la validité de la méthode.

Contrairement aux matériaux précédents, le composite Verre/Epoxyde ne peut être considéré comme étant homogène sur toute la surface insonifiée par le transducteur ( $\phi=19,05 \mathrm{~mm})$. L'interprétation des résultats reste plus délicate. Bien que présentant des variations plus importantes que pour les matériaux précédents, la célérité et l'épaisseur sont convenables. Les deux autres grandeurs ainsi que leurs variations laissent penser que le matériau est fortement inhomogène. Une étude en faisceau focalisé permettrait de suivre ces caractéristiques dans le plan, et d'en faire une cartographie..

\section{4 - CONCLUSION ET PERSPECTIVES.}

Nous avons développé une méthode simple de mesure simultanée de la vitesse de phase, de l'atténuation des ondes ultrasonores, de l'épaisseur et de la masse volumique de plaques épaisses. Les résultats obtenus en faisceau plan montrent qu'elle est utilisable pour tout matériaux composites à matrice polymérique..

La suite de cette étude consiste à étendre cette méthode au faisceau focalisé dans le but de réaliser des images des précédentes caractéristiques de zones endommagées. Des résultats préliminaires sur les mesures de vitesse et d'épaisseur, montrent que la démarche du paragraphe 2.1 peut être adoptée. Les mesures des deux autres grandeurs nécessitent une adaptation en cours de développement.

\section{Références :}

[1] Tsukahara Y., "Attenuation measurement in thin plates by ultrasonic spectroscopy", Jap. Jour. of Appl. Phys. 27-1 (1988) $58-60$.

[2] Haines N.F., Bell J.C. and McIntyre P.J., "The application of broadband ultrasonic spectroscopy to the study of layered media", J. Acoust. Soc. Am. 64 (6) (1978) 1645 - 1651.

[3] Pialucha. T. Guyott C.C.H. and Cawley P., "Amplitude spectrum method for the measurement of phase velocity", Ultrasonics 27 (1989) $270-279$.

[4] Kinra V.K., Dayal V., "A new technic for ultrasonic non destructive evaluation of thin specimen", Exp. Mech. Sept (1988) $288-297$.

[5] Kinra V.K., Zhu C., "Ultrasonic non destructive evaluation of thin (sub-wavelength) coating", J. Acoust. Soc. Am.93 (5) (1993) $2454-2467$.

[6] Hsu D.K. and Hugues M.S. , "Simultaneous ultrasonic velocity and simple thickness measurement and application in composites", J. Acoust. Soc. Am. 92 (2) (1992) $669-675$.

[7] DanieI I.M., Wooh S.C., Komsky I., "Characterisation of porosity in thick Graphite/Epoxy composites", QNDE 10B (1991) $1607-1614$.

[8] Dayal V., "An automated simultaneous measurement of thickness and wave velocity by ultrasound", Exp. Mech. Sept (1992) $197-202$.

[9] Hosten B., Barrot A. et Roux I., "Interféromètrie numérique ultrasonore pour la détermination de la matrice de raideur des matériaux composites", .Acustica 53 (1983) $212-217$. 